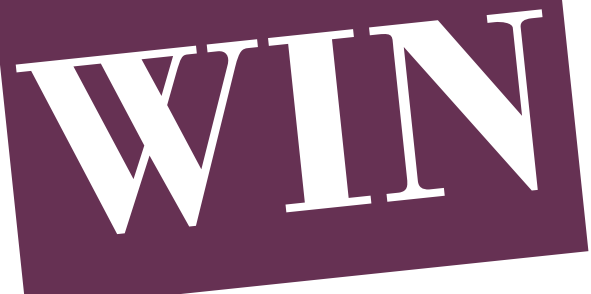

\section{a magnum of Laurent-Perrier Champagne!}

Rearrange the letters in the purple squares to find the secret word (8). Complete the answer form and send to the following address by 28 January 201 1: Vital,

BDJ Editorial, NPG, 4-6 Crinan Street, London, N1 9XW.

The answers and name of the prize winner will be

\begin{tabular}{|c|c|c|c|c|c|c|c|c|c|c|}
\hline A & $\mathrm{H}$ & A & D & S & $T / R$ & A & $\mathrm{N}$ & G & $\mathrm{E}$ & announced on \\
\hline & $\mathrm{M}$ & & $E$ & & 0 & & $E$ & & $\mathrm{~N}$ & h 2011 , \\
\hline R & $\mathrm{E}$ & $\mathrm{E}$ & $\mathrm{L}$ & & $0 . \mathrm{N}$ & $M$ & A & $\mathrm{N}$ & $\mathrm{D}$ & the sprin \\
\hline & & & $\overline{\mathrm{T}}$ & & & & $R$ & & $E$ & of Vital is \\
\hline C & L & M & A & & $\mathrm{N}$ & $\begin{array}{ll}\mathrm{v} & 0 \\
\end{array}$ & S & $\mathrm{E}$ & D & \\
\hline & E & & & & C & & & & & \\
\hline & $\mathrm{E}$ & & $U$ & & $\mathrm{E}$ & $\mathrm{N}$ & $\mathrm{N}$ & & $\mathrm{S}$ & \\
\hline & & & & & & & A & & $\mathrm{T}$ & autumn \\
\hline L & $U$ & & D & D & $\mathrm{E}$ & 0 & T & $E$ & $\mathrm{E}$ & $\mathrm{Sec}$ \\
\hline U & 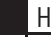 & & A & & I & & U & & $\mathrm{E}$ & left). The secret \\
\hline $\mathrm{C}$ & $\mathrm{H}$ & $\mathrm{P}$ & $\mathrm{T}$ & 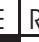 & $\underline{S}$ & $\mathrm{~T}$ & $\mathrm{R}$ & A & $P$ & word was CAVIT) \\
\hline & & & & & & & A & & $\mathrm{L}$ & \\
\hline & & & & & & & & & & \\
\hline
\end{tabular}

\section{JUST FOR FUN - WORDSEARCH}

When you have found all of the words in the above list, the remaining letters read from top to bottom will spell out a type of dentistry (12).

\begin{tabular}{|c|c|c|c|c|c|c|c|c|c|}
\hline T & R & E & A & T & M & E & N & T & F \\
\hline I & M & T & O & O & T & H & O & C & E \\
\hline P & T & A & L & E & S & L & T & A & A \\
\hline A & N & N & A & E & L & C & R & R & R \\
\hline G & N & I & P & E & E & K & O & E & F \\
\hline M & E & D & I & A & T & O & H & E & R \\
\hline Q & L & R & F & A & I & R & Y & R & E \\
\hline D & S & O & C & I & A & L & O & S & E \\
\hline T & G & O & I & M & P & L & A & N & T \\
\hline R & E & C & O & R & D & O & H & E & Y \\
\hline
\end{tabular}

Taken from this issue of Vital... TREATMENT COORDINATE HORTON RECORD KEEPING FEARFREE

SOCIAL

MEDIA

CLEAN

CAREERS

TOOTH

TALES

IMPLANT

FAIRY

Autumn issue hidden word: Inspiring

QDT OHE

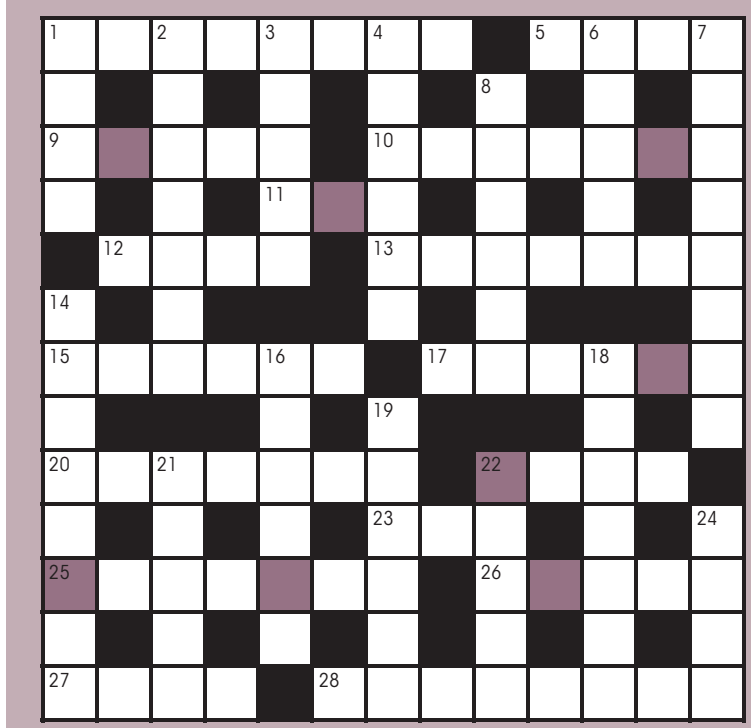

\section{Across}

1 Rush of frightened animals (8)

5 Corrosive substance (4)

9 Interior design (5)

10 Root vegetable (7)

11 Spinning toy (3)

12 Diminutive (4)

13 Implore (7)

15 Take no notice of (6)

17 Early spring flower (6)

20 Stutter (7)

22 Intense anger (4)

23 Ventilate (3)

25 Dirty washing (7)

26 Speak in public (5)

27 Always (4)

28 Gut feeling (8)

- LAST NAME

ADDRESS

I

POSTAL CODE

EMAIL

JOB TITLE

SECRET WORD

1 If you prefer not to be contacted for purposes other than this competition please tick this box.
Down

1 Mixer drink (4)

2 Sale of lots (7)

3 Political group (5)

4 Smartly dressed (6)

6 Small boat (5)

7 Act as a substitute (8)

8 Author (6)

14 Disperse in water (8)

16 Cure (6)

18 Sure (7)

19 Wax colouring pencil (6)

21 Treat badly (5)

22 Ice crystals forming a white deposit (5)

24 Amount owed (4)

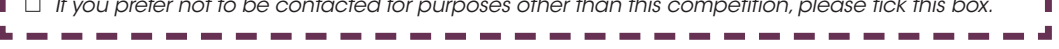

